\title{
Editorial: advanced semantic and social multimedia technologies for future computing environment
}

\author{
Seungmin Rho • Damien Sauveron • \\ Konstantinos Markantonakis
}

Published online: 24 May 2012

(C) Springer Science+Business Media, LLC 2012

\section{Introduction}

With the popularity of multimedia data on the internet, efficiently accessing and managing the explosively growing multimedia content from large-scale multimedia database has become essential. During the last decades, extensive research efforts have been dedicated to automatic multimedia analysis and processing, such as acquisition, generation, storage, and retrieval of large-scale multimedia information. However, despite these techniques already provide promising results, there are still several key difficulties such as semantic gap and user gap in multimedia analysis/processing and annotation/tagging, respectively. The aim of this special issue is to provide an overview of emerging research challenges in Semantic Multimedia and to help reducing the Semantic and User Gap between low-level content descriptions of multimedia and the semantics in high-level human interpretations of audiovisual media. Semantic Web and multimedia community researchers are encouraged to join the workshop to benefit from the presentation of results from multimedia based research, which demonstrate important multimedia applications which could be enhanced by Semantic Web technologies.

We have received 48 manuscripts. After the pre-review process, 32 manuscripts were selected for the first review. Seventeen manuscripts were finally selected for this special issue after the first, second review processes. Each manuscript selected was blindly reviewed by at least three reviewers consisting of guest editors and external reviewers.

\footnotetext{
S. Rho $(\bowtie)$

Division of Information and Communication, Baekseok University, Cheonan, South Korea e-mail: smrho@bu.ac.kr
}

D. Sauveron

University of Limoges, Limoges, France

e-mai: damien.sauveron@unilim.fr

K. Markantonakis

Royal Holloway, University of London, London, UK

e-mail: K.Markantonakis@rhul.ac.uk 
In the following section, we present a brief overview on each manuscript. In the last section, we conclude the paper with some observations and future work.

\section{Semantic and social multimedia systems}

The first paper entitled "Skin feature extraction and processing model for statistical skin age estimation," by Young-Hwan Choi et al. [1] proposes a new scheme for a self-diagnostic application that can estimate the actual age of the skin on the basis of the features on a skin image. In accordance with dermatologists' suggestions, proposed scheme examined the length, width, depth, and other cell features of skin wrinkles to evaluate skin age. Using highly developed image processing method, the proposed scheme could glean detailed information from the surface of the skin. The scheme uses the extracted information as features to train a support vector machine (SVM) and evaluates the age of a subject's skin. Evaluation of proposed scheme showed that it was more than $90 \%$ accurate in the analysis of the skin age of three different parts of the body: the face, neck, and hands.

The second paper entitled "Multi-Agent Based Middleware for Protecting Privacy in IPTV Content Recommender Services," by Mohamed et al. [3] presents their efforts to design an agent based middleware that enables the end-user to use IPTV content recommender services without revealing his sensitive profile information to that service or any third party involved in this process. The proposed middleware preserves users' privacy when using the recommendation service, Moreover it permits private sharing of data among different users in the network. Also, the proposed solution relies on a distributed multi-agent architecture involving local agents running on the end-user set up box to implement a two stage concealment process in order to conceal the local profiles of end-users with similar preferences.

Another paper in this special issue "A Multi-level Depiction Method for painterly rendering based on visual perception cue," by Lee et al. [13] proposes a method to increase the level of detail (LOD) in brushstrokes within areas of interest improved the realism of painterly rendering. They also segmented an image into areas with similar levels of saliency using a modified quad-tree and used each of those segments to control the brush strokes during rendering. They simulate real oil painting steps based on saliency information.

The fourth paper entitled "Image Recoloring Using Linear Template Mapping," by Seo et al. [15] propose an artistic recoloring method that enhance the perception of complex scenes, while preserving the visual quality of the original image. They also propose an algorithm which employs the template which is simplified representation of the colors whose color template is selected based on the Munsell color and are designed on the $\$ a^{\wedge *} b^{\wedge *} \$$ chromatic plane of the CIE $\$ \mathrm{~L}^{\wedge *} \mathrm{a}^{\wedge *} \mathrm{~b}^{\wedge *} \$$ color space.

The fifth paper entitled "CPU-Based Speed Acceleration Techniques for Shear Warp Volume Rendering," by Jeong et al. [2] proposes two techniques that can solve such defects without degrading rendering speed. One technique concentrates on enhancing image quality and decreasing memory consumption without reducing rendering speed, by making direct access to the memory space where initially loaded volume data is stored. The other technique concentrates on accelerating rendering speed and decreasing preprocessing time, by creating only one run-length-encoded volume and by combining non-photorealistic rendering techniques with shear-warp algorithm.

Next paper entitled "An Analysis of Content-Based Classification of Audio Signals using a Fuzzy C-Means Algorithm," by Kim et al. [4] presents an efficient content-based audio classification approach to classify audio signals into broad genres using a fuzzy c-means (FCM) 
algorithm. They also analyze different characteristic features of audio signals in time, frequency, and coefficient domains and select the optimal feature vector by employing a noble analytical scoring method to each feature. They utilize an FCM-based classification scheme and apply it on the extracted normalized optimal feature vector to achieve an efficient classification result..

The seventh paper entitled "SOM and Fuzzy based Color Image Segmentation," by Jaffar et al. [9] presents a modified version of fuzzy c-means (FCM) algorithm that incorporates spatial information into the membership function for clustering of color images A progressive technique based on SOM is used to automatically find the number of optimal clusters.

The eighth paper entitled "A hierarchical dense deformable model for 3D face reconstruction from skull," by Hu et al. [7] presents a hierarchical dense deformable model for automatic 3D face reconstruction from skull. Then a non-rigid dense mesh registration algorithm is presented to align all the samples in point-to-point correspondence. Based on the aligned samples, using principle component analysis technique, a global deformable model is constructed, and three local models are constructed from the segmented patches of the eye, nose and mouth. For a given skull, the globe and local deformable models are iteratively matched with it, and the reconstructed facial surface is obtained by fusing the globe and local reconstruction results. To validate the presented method, a new measurement is also defined in the coefficient domain of a face deformable model.

Another paper in this special issue, entitled "Capturing the functionality of Web services with functional descriptions," by Ruben Verborg et al. [17] develop a service description format called RESTdesc that focuses on exposing the functionality of Web services in a machineprocessable way. RESTdesc combines technology from the Web of Services, namely the REST architecture, with concepts of the Semantic Web, namely its logically sound foundation and expressive power. They illustrate how RESTdesc can be used in a multimedia use case to enable goal-driven composition using automated service discovery and consumption.

The tenth paper entitled "Integrated pricing and capacity decision for a telecommunication service provider," by Lee et al. [5] studies a price and capacity decision for a telecommunication service provider that differentiates its products based on price and quality of service to maximize revenue. They first develop a mathematical model to determine the optimal product prices and capacities necessary for maximizing total profit and then consider a case where a service provider can increase or decrease the capacity marginally, and compute the optimal price and optimal capacity.

The eleventh paper entitled "Using Augmented Reality for Students Health - Case of Combining Educational Learning with Standard Fitness," by Hsiao et al. [6] analyzes seriousness of the problem in Taiwan as an example and designed their new AR based combining exercises with the learning lessons to improve students' health whilst they continue to learn and test their other academic lessons.

Next paper entitled "Designing a Social Learning Content Management System based on Learning Objects," by Kim et al. [11] investigates a social learning content management system. According to the development of Web2.0, in the field of technology-enhanced learning, Learning Objects are the key paradigm for developing and sharing digital educational resources. Especially, depending on the spread of social networking services and social media tools, individuals was the owner to production and consumption of content. In addition, individuals have learned the value of content while sharing over the network. To this end, this paper is of significance in that it enables the transformation of social resources into learning objects based on content generation model.

The thirteenth paper entitled "Practical Service Level Agreement Negotiation Scheme for Multicast Service in WiMAX," by Lee et al. [14] suggests a novel metric which is named as channel efficiency (MCE) factor in order to give an insight of multicast efficiency. In 
addition, they proposed the practical SLA negotiation scheme with low complexity based on proposed novel metric.

Next paper entitled "The effects of social network properties on the acceleration of fashion information on the web," by Kim et al. [16] suggests the network features formed the in the internet fashion information community as variables influencing the acceptance of word-of-mouth information and analyze the actual effects in an empirical way. To do so, they conducted a social network analysis and investigated the features of the network spread patterns involving fashion information shared between community members. Then, they set up the network variables as factor influencing the acceptance of fashion information in the internet and, including these variables, conducted a significance test on the impact of the informational properties and individual characteristic variables.

The fifteenth paper entitled "A High Performance Parallel DCT with OpenCL on Heterogeneous Computing Environment," by Choi et al. [12] presents a high performance parallel implementation of 2D DCT on this heterogeneous computing environment. For this purpose, Intel TBB (threading building blocks) and OpenCL (Open Compute Language) are utilized for task- and data-parallelism, respectively.

Next paper entitled "A Practical Use of Learning System using User Preference in Ubiquitous Computing Environment," by Hong et al. [8] implements a u-learning system that considers the learning level and preferences of each learner. The system provides information about the student's preferred learning section and difficulty level of learning contents and indicates the areas that may require additional study (based on the educational history of the student), thus allowing students to set up an optimized learning environment.

The last paper entitled "Group Affinity based Social Trust Model for an Intelligent Movie Recommender System," by Park et al. [10] proposes an intelligent movie recommender system with a social trust model and the proposed system is based on a social network for analyzing social relationships between users and generated group affinity values with user profiles.

\section{Conclusion}

Finally, our special thanks go to Prof. Borko Furht and all editorial staffs for their valuable supports throughout the preparation and publication of this special issue. We would like to thank all authors for their contributions to this special issue. We also extend our thanks to the following external reviewers for their excellent help in reviewing the manuscripts.

\section{References}

1. Choi Y-H, Tak Y-S, Rho S, Hwang E (2012) Skin feature extraction and processing model for statistical skin age estimation. Multimed Tools Appl. doi:10.1007/s11042-011-0987-7

2. Choi K, Jo S, Lee H, Jeong C (2012) CPU-based speed acceleration techniques for shear warp volume rendering. Mutimed Tolls Appl. doi:10.1007/s11042-012-1010-7

3. Elmisery AM, Botvich D (2012) Multi-agent based middleware for protecting privacy in IPTV content recommender services. Multimed Tools Appl. doi:10.1007/s11042-012-1067-3

4. Haque MA, Kim J-M (2012) An analysis of content-based classification of audio signals using a fuzzy Cmeans algorithm. Multimed Tools Appl. doi:10.1007/s11042-012-1019-y 
5. Hong K-S, Lee C (2012) Integrated pricing and capacity decision for a telecommunication service provider. Multimed Tools Appl. doi:10.1007/s11042-012-1030-3

6. Hsiao K-F (2012) Using augmented reality for students health — case of combining educational learning with standard fitness. Multimed Tools Appl. doi:10.1007/s11042-011-0985-9

7. Hu Y, Duan F, Yin B, Zhou M, Sun Y, Wu Z, Geng G (2012) A hierarchical dense deformable model for 3D face reconstruction from skull. Multimed Tools Appl. doi:10.1007/s11042-012-1005-4

8. Jeong H-Y, Hong B-H (2012) A practical use of learning system using user preference in ubiquitous computing environment. Multimed Tools Appl. doi:10.1007/s11042-012-1026-Z

9. Khan A, Arfan Jaffar M, Choi T-S (2012) SOM and fuzzy based color image segmentation. Multimed Tools Appl. doi:10.1007/s11042-012-1003-6

10. Kim M, Park SO (2011) Group affinity based social trust model for an intelligent movie recommender system. Multimed Tools Appl. doi:10.1007/s11042-011-0897-8

11. Kim KR, Moon NM (2012) Designing a social learning content management system based on learning objects. Multimed Tools Appl. doi:10.1007/s11042-012-1014-3

12. Kim CG, Choi YS (2012) A high performance parallel DCT with openCL on heterogeneous computing environment. Multimed Tools Appl. doi:10.1007/s11042-012-1028-X

13. Lee H, Seo S, Ryoo S, Ahn K, Yoon K (2012) A Multi-level Depiction Method for painterly rendering based on visual perception cue. Multimed Tools Appl. doi:10.1007/s11042-012-1036-X

14. Lee J, Lee JM, Choi SG, Choi JK (2012) Practical service level agreement negotiation scheme for multicast service in WiMAX. Multimed Tools Appl. doi:10.1007/s11042-012-0998-Z

15. Seo SH, Park YS, Ostromoukhov V (2012) Image recoloring using linear template mapping. Multimed Tools Appl. doi:10.1007/s11042-012-1024-1

16. Song K, Hwang S, Kim Y, Kwak Y (2012) The effects of social network properties on the acceleration of fashion information on the web. Multimed Tools Appl. doi:10.1007/s11042-012-1068-2

17. Verborgh R, Steiner T, Van Deursen D, De Roo J, Van de Walle R, Gabarró Vallés J (2012) Capturing the functionality of Web services with functional descriptions. Multimed Tools Appl. doi:10.1007/s11042012-1004-5

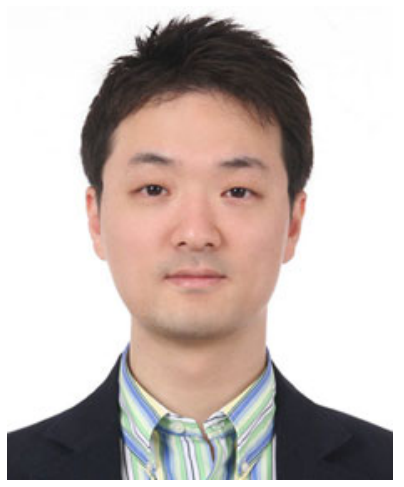

Dr. Seungmin Rho Received his MS and PhD Degrees in Computer Science from Ajou University, Korea, in Computer Science from Ajou University, Korea, in 2003 and 2008, respectively. In 2008-2009, he was a Postdoctoral Research Fellow at the Computer Music Lab of the School of Computer Science in Carnegie Mellon University. He was a Research Professor at School of Electrical Engineering in Korea University during 20092011. He is currently a faculty of the division of Information and Communication in Baekseok University. His 
research interests include database, music retrieval, multimedia systems, machine learning, knowledge management and intelligent agent technologies. He has been a reviewer in Multimedia Tools and Applications (MTAP), Journal of Systems and Software, Information Science (Elsevier), and Program Committee member in over 10 international conferences. He has published 25 papers in journals and book chapters and 30 in international conferences and workshops. He is listed in Who's Who in the World.

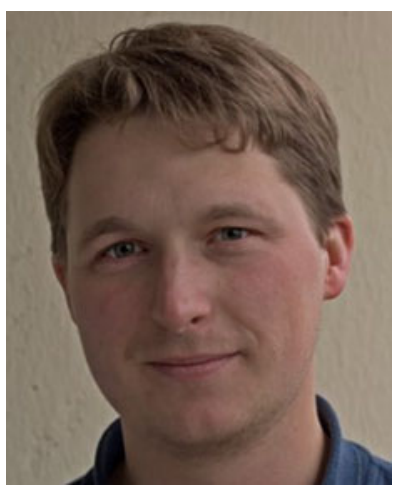

Dr. Damien Sauveron Is an assistant Professor at the XLIM (UMR 6172 University of Limoges/CNRSFrance) laboratory since $09 / 2004$ and he got a permanent position in 09/2006. In 2008, he has been elected for 4 years as member of the scientific council of the University of Limoges. He was member of the DMI department (Département Mathématiques Informatique/Mathematics and Computer Sciences Department-led by Prof. Moulay Barkatou) of XLIM. He was also involved in the SeFSI program (Sécurité et Fiabilité des Systèmes d'Information/Security and Safety of Information Systems - SeFSI is a transverse initiative of XLIM led by Prof. Jean-Pierre Borel). For the 09/2006-09/2008 period, the University of Limoges and the Limousin Region have decided to fund my research. He obtained my Ph.D. at the University Bordeaux 1 (France) in December 2004. From 03/09/2001 to 02/09/2004, He worked on the Java Card security in the ITSEF (Information Technology Security Evaluation Facilities) of SERMA Technologies. From 01/02/2006 to 31/03/2006, He was invited researcher at the ISG-SCC (Information Security Group-Smart Card Centre) of the Royal Holloway, University of London (RHUL). Then, from the $01 / 04 / 2006$ to $10 / 08 / 2006$ I was in a postdoctoral position at the ISG-SCC of the RHUL. From 09/2004: Assistant Professor in the "Security of Information" team at the University of Limoges (France). He also work with the "Distributed Systems and Objects" team of Professor Serge Chaumette at the LaBRI (Laboratoire Bordelais de Recherche en Informatique) on many topics.

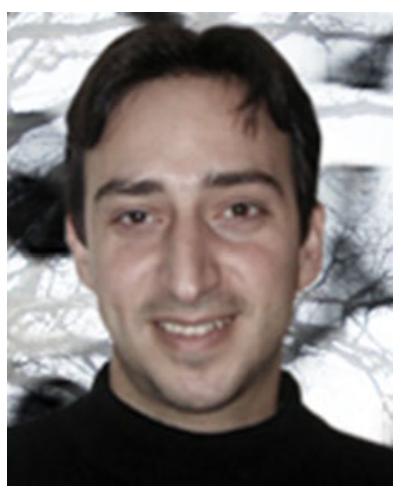

Dr. Konstantinos Markantonakis B.Sc. (Lancaster University), M.Sc., Ph.D. (London) received his BSc (Hones) in Computer Science from Lancaster University in 1995, his MSc in Information Security in 1996, his 
$\mathrm{PhD}$ in 2000 and his MBA in International Management in 2005 from Royal Holloway, University of London. He is currently a Reader in the Information Security Group. His main research interests include smart card security and applications, secure cryptographic protocol design, Public Key Infrastructures, key management, mobile phone security, embedded systems. Since completing his $\mathrm{PhD}$, he has worked as an independent consultant in a number of information security and smart card related projects. He has worked as a Multiapplication smart card Manager in VISA International EU, responsible for multi-application smart card technology for southern Europe. More recently, he was working as a Senior Information Security Consultant for Steer Davies Gleave, responsible for advising transport operators and financial institutions on the use of smart card technology. He is also a member of the IFIP Working Group 8.8 on Smart Cards. He continues to act as a consultant on a variety of topics including smart card security, key management, information security protocols, mobile devices, smart card migration program planning/project management for financial institutions, transport operators and technology integrators. 